\title{
Perceived angle of oscillatory motion ${ }^{1}$
}

WILLIAM EPSTEIN, UNIVERSITY OF RANSAS

GUNNAR JAMSSON AND GUNNAR JOHANSSON, UNIVERSITY OF UPPSALA, SWEDEN

The general background of these experiments was the fact, known from e.g. trapezoidal window experiments, that rotary motion under certain conditions is perceived as oscillation. The aim was to identify the variables that determine the perceived angle of this oscillatory motion. Different shapes and different methods of generating the stimulation were used. No effect was obtained when varying the degree of trapezoidality of trapezoids, the location of the axis of rotation, the size of the stimulus pattern and the speed of the change. Both the occurrence and the perceived angle of oscillation was effected, however, by the width-height ratio of the stimulation, a decreasing ratio giving increasing occurrence of oscillation and decreasing perceived angle.

Under certain conditions, objectively rotating forms are perceived to oscillate instead of rotating. The form appears to swing back and forth, the direction of movement appearing to reverse itself twice during each $360^{\circ}$ rotation. The best known example is Ames' (1951) rotating trapezoidal window. However, illusory oscillation has been exhibited with a variety of forms, including several nonrepresentative, irregularly contoured forms (Pastore, 1952; Day \& Power, 1965). The explanation of the oscillation phenomenon continues to be debated (Ames, 1951; Pastore, 1952; Kilpatrick, 1953; Graham, 1963; Day \& Power, 1965, Epstein, 1967).

As a rule, investigations of the phenomenon have been concerned with the gross aspects. Typically, the dependent variable has been the occurrence of perceived oscillation per se, in contrast to rotation. We think it will be valuable to focus on the finer aspects of the phenomenon. Information about these details may help us to decide between the alternative explanations.

The objective of this paper is to describe five experiments that sought to identify the variables that determine the percelved angle or amplitude of oscillation. Experiments 1 and 2 used trapezoidal forms as standards. Experiments 3-5 employed a variety of different forms. Experiment 1 investigated the effects of varying the degree of trapezoidality, i.e., the extent to which the angles deviate from $90^{\circ}$. Experiment 2 studied the effects of varying the location of the vertical axis of rotation. Experiment 3 explored the effects of variation of the width of the standard, when height was constant. Experiment 4 studied the same question as Experiment 3 , but with a new experimental arrangement. Experiment 5 examined the effects of variations of shape, absolute size, and velocity of the standard.

\section{EXPERIMENT 1}

The purpose of Experiment 1 was to determine whether changes in the perceived angle of oscillation would result from variations of trapezoidality.

\section{Method}

Standards. The standards were three skeletal trapezoids made from wire and painted with fluorescent paint. Each trapezoid was $114 \mathrm{~mm}$ wide and had a long vertical side of $114 \mathrm{~mm}$. The size of the small angle was $67.6^{\circ}, 75.4^{\circ}$, and $84.7^{\circ}$ for standards $\mathrm{A}, \mathrm{B}$, and $\mathrm{C}$, respectively.

Apparatus. The standards were rotated mechanically at the speed of $6 \mathrm{rpm}$. Illumination was provided by two ultraviolet black lights that were positioned on either side of the standard. S tracked the perceived path of movement by swinging a crank back and forth to simulate the apparent movement of the standard. The movements of the crank were recorded on a dual channel recorder, which provided an exact record of S's responses.

Viewing conditions. S sat in a normally illuminated room and viewed the standards monocularly through a rectangular window from a distance of approximately $1.75 \mathrm{~m}$. The room containing the standard was dark, so that only the standard was visible.

Instructions. Experiment $1 \mathrm{a}-\mathrm{S}$ was informed that rotating forms would be presented, but that oscillation was frequently perceived under the special conditions of observation that prevailed in the experiment. $S$ was instructed to track the perceived oscillation.

Experiment $1 \mathrm{~b}$-The instructions of Experiment 1a were revised to stress the importance of accurate tracking. E explained that the aim of the experiment was to obtain information about the angle of oscillation and, therefore, S should give particular attention to the turning points.

Subjects. The Ss were 24 undergraduate psychology students, 12 in each experiment.

Procedure. S judged each of the three standards. Each standard was exposed continuously until E secured records of 10 perceived oscillations. This meant that the trial durations varied for different Ss and often for the different standards with the same $\mathrm{S}$.

\section{Results}

The results are summarized in Table 1. In Experiment $1 \mathrm{a}$, the mean angle of oscillation for the three standards combined was $126^{\circ}$. There were 
small differences between the standards, but these are not significant. There was considerable variability among the judgments for each standard. The grand mean angle of oscillation for all standards in Experiment $1 \mathrm{~b}$ was $98^{\circ}$. Experiments $1 \mathrm{a}$ and $1 \mathrm{~b}$ differed only in the phrasing of the instructions. Therefore, we may attribute the difference between the mean judged oscillation to the greater concern with accurate matching in Experiment 1b. Again, there were small differences between the standards, but these were not significant. The combined means for Experiments $1 \mathrm{a}$ and $1 \mathrm{~b}$ are almost identical for the three standards, $\mathrm{A}=112^{\circ}, \mathrm{B}=111^{\circ}$, and $\mathrm{C}=113^{\circ}$.

\section{Conclusions}

There is no evidence found that variations of perceived angle of oscillation are associated with variations of trapezoidality. Since a rotating rectangle will appear to rotate, it would seem that a small deviation from $90^{\circ}$ (not greater than $5.3^{\circ}$ ) of the angles between the sides results in an all-ornone shift from rotation to oscillation.

\section{EXPERIMENT 2}

The most common arrangement for demonstrating the oscillation phenomenon is rotation on a vertical axis that bisects the standard. This was the arrangement in Experiment 1. In Experiment 2 the location of the axis of rotation was varied and judgments of oscillation were obtained.

Standards. Trapezoid A of Experiment 1 was used. Five axis locations were tested: (1) central location as in Experiment 1; axis coinciding with the short (2) and long (3) sides; axis located on the left (4) and right (5) of the central location, midway between the center and the side.

Subjects. Twelve new students from the same population served as Ss.

Procedure. The apparatus and procedure were the same as in Experiment 1a. Each $S$ judged all five standards.

\section{Results}

Three Ss reported only rotation when the axis was continuous with the shorter side of the trapezoid. One of these three Ss also reported rotation for the central location and two other locations. Since our concern is with perceived oscillation we have ex-

Table 1. Perceived Angle of Oscillation for the Three Trapezoids of Experiment 1

\begin{tabular}{crrrrrrr} 
& \multicolumn{9}{c}{ Standard Trapezoid } \\
Experiment & A. $67.6^{\circ}$ & \multicolumn{2}{c}{ B. $75.4^{\circ}$} & \multicolumn{2}{c}{ C. $84.7^{\circ}$} \\
\hline $1 \mathrm{o}$ & $\mathrm{M}$ & SD & M & SD & M & SD \\
\hline $1 \mathrm{~b}$ & $131^{\circ}$ & $37^{\circ}$ & $127^{\circ}$ & $35^{\circ}$ & $121^{\circ}$ & $34^{\circ}$ \\
Combined & $93^{\circ}$ & $19^{\circ}$ & $95^{\circ}$ & $14^{\circ}$ & $107^{\circ}$ & $22^{\circ}$ \\
\hline
\end{tabular}

cluded this latter. S, and we have computed medians for the remaining $11 \mathrm{Ss}$. The median perceived angle of oscillation was $146^{\circ}, 141^{\circ}, 153^{\circ}, 147^{\circ}$, and $133^{\circ}$ for axis locations $1,2,3,4$, and 5 , respectively. These differences are not significant.

\section{Conclusions}

There was no evidence that varying the location of the vertical axis of rotation results in different amounts of perceived oscillation. One feature of the results merits additional comment. Relocating the axis from the central position to the side position did not affect the oscillation phenomenon significantly. This finding suggests that reciprocal variations of the visual angle of the two vertical sides, which occurs when the axis is centrally located, is not an essential condition of the oscillation phenomenon. When the axis of rotation is continuous with one of the sides, rotation results in transformations of the visual angle of the opposite side only. The visual angle subtended by this side expands and contracts, while at the same time, the visual angle subtended by the side that is continuous with the axis remains constant throughout the course of rotation.

\section{EXPERIMENT 3}

Does the width of the form affect the amplitude of perceived oscillation? It will be noted that the variations introduced in Experiments 1 and 2 did not affect the width of the forms, which remained the same for all conditions. Experiment 3 bears on this question. And although the standards of this experiment were not trapezoids, we believe that the findings, taken together with those of the two succeeding experiments, may be generalized for the case of trapezoidal forms.

\section{Method}

Standards. The standards were five skeletal fluorescent figures, a circle and four ellipses. The circle had a diameter of $11.5 \mathrm{~cm}$. The ellipses had their vertical axes equal to the diameter of the circle. The horizontal dimension was varied to produce ellipses with axis ratios of $0.80,0.60,0.40$, and 0.20 . The standards were rotated along the bisecting vertical axis.

Instructions. The instructions were open-ended, merely instructing $S$ to track the movement of the standard, without emphasizing oscillation.

Apparatus. The apparatus of Experiments 1 and 2 was modified to allow $S$ to simulate a full $360^{\circ}$ rotation.

Procedure. The procedure was the same as in the earlier experiments, except that the five standards were presented for free inspection prior to beginning the experimental trials. $S$ was informed that each of the standards would be shown at least 
Table 2. Amplitude of Perceived Motion for a Circle and Four Ellipses of Varying Width: Experiment 3

\begin{tabular}{|c|c|c|c|c|c|}
\hline \multirow[b]{2}{*}{ Subject } & \multirow{2}{*}{$\frac{\text { Circle }}{1.0}$} & \multicolumn{4}{|c|}{ Standard Ellipses } \\
\hline & & 0.80 & 0.60 & 0.40 & 0.20 \\
\hline 1 & Rotation & Rotation & Rotation & Rotation & Rotation \\
\hline 2 & Rotation & Rotation & $119^{\circ}$ & $90^{\circ}$ & $52^{\circ}$ \\
\hline 3 & Rotation & $110^{\circ}$ & $110^{\circ}$ & $74^{\circ}$ & $56^{\circ}$ \\
\hline 4 & Rotation & $142^{\circ}$ & $137^{\circ}$ & $99^{\circ}$ & $68^{\circ}$ \\
\hline 5 & Rotation & Rotation & $180^{\circ}$ & $141^{\circ}$ & $59^{\circ}$ \\
\hline 6 & Rotation & Rotation & Rotation & $90^{\circ}$ & $61^{\circ}$ \\
\hline 7 & $158^{\circ}$ & $131^{\circ}$ & $90^{\circ}$ & $65^{\circ}$ & $52^{\circ}$ \\
\hline 8 & Rotation & $160^{\circ}$ & $97^{\circ}$ & $108^{\circ}$ & $105^{\circ}$ \\
\hline 9 & Rotation & Rotation & Rotation & $92^{\circ}$ & $110^{\circ}$ \\
\hline 10 & Rotation & Rotation & $180^{\circ}$ & Rotation & $168^{\circ}$ \\
\hline 11 & Rotation & $169^{\circ}$ & $144^{\circ}$ & Rotation & $106^{\circ}$ \\
\hline 12 & $180^{\circ}$ & $180^{\circ}$ & $168^{\circ}$ & $168^{\circ}$ & $168^{\circ}$ \\
\hline 13 & Rotation & Rotation & Rotation & Rotation & Rotation \\
\hline 14 & Rotation & Rotation & Rotation & Rotation & $96^{\circ}$ \\
\hline 15 & Rotation & $160^{\circ}$ & Rotation & $130^{\circ}$ & $100^{\circ}$ \\
\hline
\end{tabular}

once during the experiment. In addition, the speed of rotation was increased to $12 \mathrm{rpm}$.

Subjects. Fifteen new Ss served in this experiment.

\section{Results}

The results are given in Table 2. The following trends appear in the group data: (1) The likelihood of perceiving oscillation is related to the width of the standard. The frequency of oscillation reports was $2,7,9,10$, and 13 for the circle and the .80 , .60 , .40, and .20 ellipses, respectively. (2) When oscillation is perceived, the angle of oscillation is related to the width of the standard. The median perceived angle was $169^{\circ}, 160^{\circ}, 137^{\circ}, 96^{\circ}$, and $82^{\circ}$ for the circle and the ellipses in order of decreasing width.

As the width of the standard is reduced, the likelihood of seeing oscillation increases and the angle of oscillation decreases.

An examination of the individual data presented in the table shows not all of the Ss conformed to the group trends. Particularly deviant were the responses of Ss 8,9 , and 12 .

\section{Conclusions}

The results suggest that the angle of percelved oscillation depends on the width of the form. Individual differences are commonly found in experimental settings that provide less information than is available under typical "natural" conditions of observation. The data in Table 2 exhibit such differences. There are at least two alternative interpretations of this variability: (1) Ss differ in their ability to extract information that is latent in the geometry of optical stimulation, and (2) Ss differ in their ability or inclination to match what they perceive. At present, it is not possible to decide between these alternatives.

\section{EXPERIMENT}

Experiment 4 reexamined the question studied in Experiment 3 with a different type of stimulation. In addition, two new forms were studied.

\section{Method}

Apparatus. Experiments 1-3 used mechanical means to present real objects that rotated in three dimensional space. In Experiments 4 and 5 we abandoned this method in favor of a method of generating stimuli artificially. This method has been used successfully in earlier studies of the perception of motion and changing form (Johansson, 1964; Johansson \& Johansson, 1967).

A block diagram of the apparatus for generating the stimuli is shown in Fig. 1. The Ss viewed a virtual image of the pattern on an oscilloscope screen and tracked the apparent movement of the image with the response device used in Experiment 3. The optical device used is described in Johansson (1964).

Standards. The standards were parallel projections of rotating half-ellipses and rectangles. The ellipses were outline figures, i.e., only the contours were presented. The rectangles were filled figures. The maximal horizontal extension (width) was varied. The height was the same for all standards. Table 3 shows the dimensions of the stimuli.

Subjects. There were $14 \mathrm{Ss}, 13$ from the same student population that provided Ss for the preceding experiments.

Procedure. The experiment began with a practice session that was designed to familiarize $S$ with the

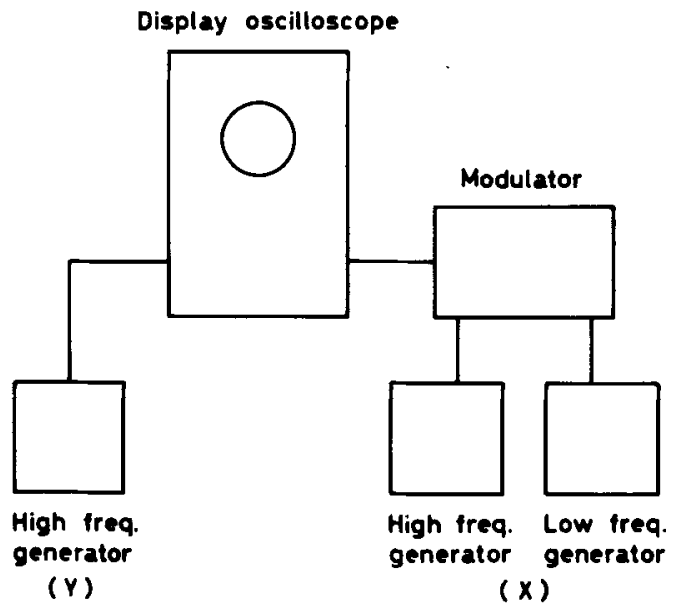

Fig. 1. Blockdiagram of the Stimulus Generator used in Experiments 4 and 5 . When generating the rectangles high frequencies with a mutually complex ratio relation were chosen. When generating ellipses both channels had the same high frequencies with a controlled phase difference. (A variable phase function generator was used.) The $\mathbf{X}$-modulator gave a change symmetrical around zero. All frequencies were sinusoidal. E observed the stimulation on a monitor oscilloscope. 
Table 3. Perceived Angle of Oscillation of the Stimulus Pattems in Experiment 4. (The means and SD of the individual means are given. $\mathbf{N}$ refers to the number of Ss having oscillation percepts. All stimuli had the $\theta$ height of $3.50^{\circ}$.)

\begin{tabular}{lcccc} 
& $\theta$ Maximum Width & $N$ & Mean & SD \\
\hline Ellipses & & & & \\
E 1 & $3.50^{\circ}$ & 11 & $146^{\circ}$ & 31 \\
E 2 & $1.74^{\circ}$ & 12 & $119^{\circ}$ & 40 \\
E 3 & $0.88^{\circ}$ & 13 & $82^{\circ}$ & 26 \\
E 4 & $0.35^{\circ}$ & 13 & $47^{\circ}$ & 23 \\
Rectangles & & & & \\
R 1 & $3.50^{\circ}$ & 10 & $155^{\circ}$ & 40 \\
R 2 & $1.74^{\circ}$ & 10 & $122^{\circ}$ & 43 \\
R 3 & $0.88^{\circ}$ & 9 & $101^{\circ}$ & 49 \\
R 4 & $0.35^{\circ}$ & 11 & $46^{\circ}$ & 20 \\
\hline
\end{tabular}

situation. One ellipse and one rectangle were selected at random from the test series, and $\mathrm{S}$ was shown how to simulate the perceived movement of the pattern. S was instructed to look at the whole pattern, and to avoid fixing his attention on a specific point of the contour. The eight stimuli described in Table 3 were presented in a different random order to each S. Each stimulus was shown for 100 sec, 20 cycles for each stimulus. The $S$ tracked the apparent motion continuously. 2

\section{Resulis}

Most of the Ss (nine) saw both rotation and oscillation with the ellipses, and all of the Ss reported both percepts with the rectangles. In addition, there were some reports of changing shape with the rectangles. By examining the continuous tracking records it was possible to determine the percentage of each trial during which rotation and oscillation were perceived. There was a general tendency for the oscillation period to increase as the width of the stimulus decreased. For example, the nine ss who reported both rotation and oscillation for the ellipses, but no shape change, produced records which gave evidence of perceived oscillation during $35 \%, 53 \%, 68 \%$, and $78 \%$ of the viewing time for patterns E 1, E 2, E 3, and E 4, respectively. This finding is compatible with the frequency data reported for Experiment 3.

Table 3 shows the perceived angle of oscillation for the eight patterns. In agreement with the results of Experiment 3, the present findings show that the angle of oscillation decreases as the width of the figure decreases.

\section{EXPERIMENT 5}

In Experiment 4 the effect of the maximum width of the stimulus patterns on perceived movement and perceived angle of oscillation was studied. However, since the height of the stimuli and the modulating frequency were held constant, the shape and the velocity of the moving curved part of the figures covaried with the width of the stimulus figures. The presence of this covariation complicates the interpretation of Experiment 4. Experiment 5 was designed to assess the relative importance of these variables. The following variables were studied: (1) Shape in maximal extension; (2) Absolute size in maximal extension; (3) Velocity of the change.

\section{Method}

Apparatus. The same apparatus as in Experiment 4 was used.

Subjects. Fifteen students of psychology served as Ss.

Stimuli. The stimuli were oscilloscope-generated parallel projections of rotating half-ellipses. These were outline figures, i.e., only the contours were presented. Table 4 shows the different stimuli.

Procedure. A brief description of the stimulus figures was given to $\mathrm{S}$. When instructing $\mathrm{S}$ regarding the response device, both oscillation and rotation were mentioned as likely percepts. A practice trial was given, and $S$ was reminded not to look steadily at any particular point but to look passively at the whole stimulus pattern.

The 11 stimuli were presented once in a randomized order. Every stimulus was shown for 100 sec, which means that stimuli 1-9 completed 20 cycles, while stimuli 10 and 11 completed 40 cycles. The $S$ responded continuously by manipulating the response device.

\section{Results}

One $S$ did not perceive rotation at any moment. Among the remaining $14 \mathrm{Ss}$, rotation percepts did occur, but oscillation percepts were far more frequent. In a few cases only oscillation was perceived. The perceived angle of oscillation is shown in Table 4. The effect of the three stimulus variables can be studied in the following way.

The effect of the shape in maximal extension is shown in the groups 1-4-7, 2-5-8, and 3-6-9. If the shape is crucial for the perceived angle of oscillation, these groups should produce nonsignificant

Table 4. Perceived Angle of Oscillation of the Stimulus Pattems in Experiment 5. (The means and SD of the individual means are given. $\mathbf{N}$ refers to the number of Ss having oscillation percepts.)

\begin{tabular}{cccccccc} 
Number & \multicolumn{2}{c}{$\begin{array}{c}\text { Stimulus } \\
\theta \text { Height }\end{array}$} & $\begin{array}{c}\theta \text { Maximum } \\
\text { Width }\end{array}$ & $\begin{array}{c}\text { Frequency } \\
\text { (cps) }\end{array}$ & $N$ & Merceived angle & SD \\
\hline 1 & $4.54^{\circ}$ & $4.54^{\circ}$ & 0.2 & 15 & $161^{\circ}$ & 25 \\
2 & $4.54^{\circ}$ & $2.28^{\circ}$ & 0.2 & 14 & $133^{\circ}$ & 26 \\
3 & $4.54^{\circ}$ & $1.14^{\circ}$ & 0.2 & 15 & $84^{\circ}$ & 24 \\
4 & $2.28^{\circ}$ & $2.28^{\circ}$ & 0.2 & 15 & $160^{\circ}$ & 35 \\
5 & $2.28^{\circ}$ & $1.14^{\circ}$ & 0.2 & 15 & $119^{\circ}$ & 32 \\
6 & $2.28^{\circ}$ & $0.57^{\circ}$ & 0.2 & 15 & $85^{\circ}$ & 47 \\
7 & $1.14^{\circ}$ & $1.14^{\circ}$ & 0.2 & 15 & $153^{\circ}$ & 30 \\
8 & $1.14^{\circ}$ & $0.57^{\circ}$ & 0.2 & 15 & $125^{\circ}$ & 29 \\
9 & $1.14^{\circ}$ & $0.29^{\circ}$ & 0.2 & 15 & $75^{\circ}$ & 27 \\
10 & $4.54^{\circ}$ & $2.28^{\circ}$ & 0.4 & 13 & $123^{\circ}$ & 35 \\
11 & $4.54^{\circ}$ & $1.14^{\circ}$ & 0.4 & 14 & $78^{\circ}$ & 29 \\
\hline
\end{tabular}


differences within the groups but significant ones between the groups. As can be seen from Table 4 , this is the result.

If the absolute size in maximal extension in one of the dimension is crucial, the same height or maximum width, respectively, would produce the same response. As can be seen from Table 4, this is not the case.

Lastly, if the velocity of the change is crucial, stimulus 10 should yield the same response as stimulus 1 , and stimulus 11 the same as stimulus 2 . This is not the case.

It seems reasonable to conclude that the shape of the stimulus determines the perceived angle. Effects of absolute size of the pattern and velocity of the change did not appear. The effects of shape conformed to the trends in the data of Experiments 3 and 4.

\section{DISCUSSION}

When a rotating figure appears to oscillate, what are the variables that determine the perceived angle of oscillation? The results of Experiments 1 and 2 gave no evidence that degree of convergence, or the location of the axis of rotation, are important, insofar as trapezoidal figures are concerned. Trapezoidal figures were selected as standard stimuli in Experiments 1 and 2 primarily because the trapezoid served as the standard in Ames' important study. In Experiments 3-5, the standards were circles, ellipses, half-ellipses, and rectangles. Experiments 3 and 4 showed that variations of width are accompanied by variations in the perceived angle of oscillation. This raised the question of whether the effects of width were due to absolute variations of size or to variations of the heightwidth ratio. Changes of height-width ratio affect the shape of the figure, whereas shape is invariant over size transformations that do not alter the height-width ratio. The results of Experiment 5 plainly favored the ratio or shape interpretation. Differences in the perceived angle of oscillation were associated with changes in the height-width ratio, but no differences accompanied changes in absolute size. Specifically, the main finding is that perceived angle of oscillation decreases as the height-width ratio deviates increasingly from unity. An explantion of our findings is best deferred until further work has been done. In our view, this work should include a detailed analysis of the proximal stimulation and experiments based on this analysis. Work is in progress to further this objective with application of the same general approach as that of Johansson (1964) and Johansson and Johansson (1967). At this juncture, we can only assert that the shape variable will have to be included in any account of the oscillation phenomenon that purports to be complete.

\section{References}

Ames. A. Visual perception and the rotating trapezoidal window Psychol. Monoar., 1951, 65, No 7 (Whole No. 324)

Day, R. H. \& Power, R. P. Apparent reversal (oscillation) of rotary motion in depth: an investigation and a general theory. Psychol. Ret., 1965, 72, 117-127

Epstein, W. Varieties oj pereeptual learning. New York: McGraw Hill, 1967

Graham, C. H. On some aspects of real and apparent visual movement. J. Opt. Soc. Amcr., 1963, 53, 1019-1025.

Kilpatrick, F. P. The Ames oscillatory effect: A reply to Pastore Psychol. Rer., 1953, 60, 76-79

Johansson, G. Perception of motion and changing form. Scand. $J$ Psychol., 1964, 5, 181-208

Johansson, G., \& Jansson, G. A model for space perception and object perception from changes in a straight line. 41 st Rep. Psychol. Lab., U. Uppsala, 1967

Pastore, N. Some remarks on the Ames oscillatory effect, Psychol. $R e r, 1952,59,319-323$

\section{Notes}

1. The experiments were conducted at the University of Uppsala. Valuable assistance was provided by Erik Börjesson, Karin Lindhagen, Sverker Runeson, and Marianne Snöborgs. During the period of this research, William Epstein was associated with the University of Uppsala as Senior Postdoctoral Fellow of the National Science Foundation.

2. Immediately following each movement trial, $\mathbf{S}$ was required to make a shape-judgment. We are omitting this phase of the experiment from our report, principally because we doubt the validity of our procedure. Inasmuch as the eight stimulus-patterns were exposed in a different order for each $S$, the effects of the shapejudgments, if any, may be assumed to be equally distributed among the patterns.

(Accepted for publication September 29, 1967. 